Sociologie et sociétés

\title{
Les milieux sociaux dans le roman canadien-français contemporain
}

\section{Jean-Charles Falardeau}

Volume 48, numéro 2, automne 2016

Sociologie narrative : le pouvoir du récit

Narrative sociology: the power of storytelling

URI : https://id.erudit.org/iderudit/1037726ar

DOI : https://doi.org/10.7202/1037726ar

Aller au sommaire du numéro

Éditeur(s)

Les Presses de l’Université de Montréal

ISSN

0038-030X (imprimé)

1492-1375 (numérique)

Découvrir la revue

Citer cet article

Falardeau, J.-C. (2016). Les milieux sociaux dans le roman canadien-français contemporain. Sociologie et sociétés, 48(2), 305-324.

https://doi.org/10.7202/1037726ar d'utilisation que vous pouvez consulter en ligne. 


\section{Les milieux sociaux dans le roman canadien-français contemporain ${ }^{1}$}

JEAN - CHARLES FALARDEAU

Département de sociologie et d'anthropologie Université Laval

L

E ROMAN EST PLUS QUE LE SIMPLE REFLET D'UNE RÉALITÉ SOCIALE. Il est une création spécifique, à partir d'éléments que l'écrivain trouve en lui-même et autour de lui. Le romancier invente des êtres et les lance dans des aventures humaines. Il pousse jusqu'à leurs limites des destinées dont il a trouvé des indices dans son expérience. Il rend explicite ce qu'il a vu comme latent; il décrit comme vraisemblable ce qu'il a pressenti comme possible; il offre comme organisé ce qu'il a observé comme diffus. Il a sous les yeux le spectacle de vies qui, comme le dit Jules Romains, «ne vont nulle part», de passions étouffées, de communications tronquées. De ces suggestions éparses, sporadiques, que lui propose la réalité, il compose, comme avec des thèmes donnés, une structure symphonique qui s'imposera à la fois comme révélation d'un inconnu et comme l'écho d'une expérience obscurément familière.

L'œuvre ainsi créée place le lecteur au centre d'un « univers », celui du romancier, qui tout à la fois n'est pas la société réelle et dérive de la société réelle. L'œuvre littéraire

1. Ce texte de Jean-Charles Falardeau a été publié pour la première fois en 1964 dans Recherches sociographiques (volume 5, nº 1-2, p. 123-144), revue dont il a été le cofondateur en 1960 à l'Université Laval. On remercie la rédactrice de la revue, Sylvie Lacombe, de permettre à Sociologie et sociétés de publier ce texte à nouveau étant donné sa pertinence pour le présent numéro thématique. 
comporte cette ambiguïté. Elle est transcription d'un monde rêvé mais elle est aussi témoignage d'un sens profond qui justifie et sous-tend ce rêve. Ce sens, le romancier l'a discerné à travers l'opacité enveloppante du tissu social qui le porte, qu'il tolère ou qu'il condamne, dont il voit les incohérences, et avec lequel, quelle que soit la distance qu'il prend avec lui, il a partie liée. Visionnaire sans doute, mais visionnaire d'une réalité qu'il est le seul à percevoir, au delà de l'écran protecteur des conventions. Visionnaire incarné dont la vision ne serait pas ce qu'elle est si les choses n'étaient pas d'abord ce qu'elles sont. L'expression littéraire peut être acceptation, cri, révolte, sublimation ou mythologie. Quel que soit son registre, elle n'a de sens qu'à partir d'une société donnée. Le roman, c'est la société rêvée, transposée, recomposée, transfigurée, refigurée, transcendée.

C'est dans cette perspective que nous abordons la littérature canadienne-française contemporaine. De quelques-uns de ses romans récents, nous voulons dégager une sorte de topographie sociale. Nous cherchons à mettre à jour le relief des univers sociaux dans lesquels les romanciers font évoluer leurs créatures imaginaires - des créatures que nous sentons souvent si étrangement près de notre mystère intérieur. Nous noterons ainsi successivement comment apparaissent dans les romans: $1^{\circ}$ les cadres géographiques d'existence collective; $2^{\circ}$ les familles; $3^{\circ}$ certaines institutions dominantes; $4^{\circ}$ des types professionnels significatifs; $5^{\circ}$ les classes sociales.

\section{LES CADRES GÉOGRAPHIQUES}

C'est un chef-d'œuvre de la vie rurale, le Trente arpents de Ringuet, qui a éliminé ou à peu près la vie rurale du roman canadien contemporain. Un certain nombre d'œuvres se déroulent encore à la campagne ou persistent à la glorifier. Elles demeurent des exceptions. À un pôle, nous trouvons les exaltations lyriques ou mythologiques d'un Félix-Antoine Savard. Au pôle opposé se groupent les œuvres plus nombreuses d'une Germaine Guèvremont, d'un Yves Thériault, d'un Bertrand Vac. La campagne, qu'elle soit une campagne authentique comme la vallée du Richelieu ou une campagne fictive comme le «nord» de Montréal, est devenue lieu de promenade, lieu d'évasion, lieu de refuge ou incarnation de nostalgies compensatrices. Rappelons la visite soudaine de la famille Lacasse de Bonheur d'occasion chez les parents de Rose-Anna, un dimanche du temps des sucres; l'escapade d'un Alexandre Chenevert; les fugues vers les Laurentides des jeunes bourgeois montréalais de Ringuet; le dimanche au lac Sergent des Lévesque, de Denis Boucher et Jean Colin dans Au pied de la Pente Douce.

Un certain nombre de romans, par contre, situent l'ensemble ou des épisodes importants de leur histoire dans un décor intermédiaire entre la campagne et la ville: le camp de bûcherons. Celui-ci est le décor d'un chapitre décisif de Pierre le magnifique de Lemelin, du long prologue du roman de Pierre Gélinas, Les vivants, les morts et les autres, de toute la sombre aventure racontée par Langevin dans Le temps des hommes. Le camp de bûcherons n'est pas choisi pour ses aspects pittoresques. Il est proprement un champ de bataille. Dans deux des trois exemples que nous venons de mentionner, le camp de bûcherons est le théâtre d'une grève ou d'un commencement de grève: des 
travailleurs en forêt exploités et jusque-là passifs sont mobilisés contre leurs patrons étrangers ou absentéistes par des entraîneurs qui ont lu Marx ou qui ont seulement commencé à étudier les sciences sociales. La forêt était jadis symbole de la terre à conquérir, de la fuite en dehors de la société, d'une lutte entre l'homme et la nature. Ce dernier retranchement a été rejoint par la société. Cette zone des refuges ultimes a été intégrée au réseau des idéologies, des institutions, des conflits d'origine industrielle et urbaine.

La très grande majorité des romans ont maintenant comme cadre un milieu urbain. Ce milieu, c'est Montréal ou Québec, c'est plus particulièrement un quartier de Montréal ou de Québec. Très souvent, aussi, c'est une petite ville de province. Les premiers grands romans de la vie urbaine qui ont fait suite à Trente arpents - Bonheur d'occasion, Au pied de la Pente Douce et Les Plouffe — ont comme personnage principal un quartier de la ville.

La topographie, les rythmes, les odeurs, les perspectives géographiques et sociales du quartier Saint-Henri de Montréal sont organiquement mêlés, dans Bonheur d'occasion, à l'existence de la famille Lacasse, de Jean Lévesque, d'Emmanuel Létourneau, de tous les sans-travail, de tous les travailleurs, de l'ensemble du petit peuple besogneux, irrité, bouillonnant qui est emmuré dans «ce village dans la grande ville» (393). ${ }^{2}$ Dès les premières pages du roman, nous prenons intensément possession du quartier à travers les impressions et la vision de Jean Lévesque:

Le train passa. Une âcre odeur de charbon emplit la rue. Un tourbillon de suie oscilla entre le ciel et le faîte des maisons. La suie commençant à descendre, le clocher de Saint-Henri se dessina d'abord, sans base, comme une flèche fantôme dans les nuages. L'horloge apparut; son cadran illuminé fit une trouée dans les voiles de vapeur; puis, peu à peu, l'église entière se dégagea, haute architecture de style jésuite. Au centre du parterre, un SacréCœur, les bras ouverts, recevait les dernières parcelles de charbon. La paroisse surgissait. Elle se recomposait dans sa tranquillité et sa puissance de durée. École, église, couvent: bloc séculaire fortement noué au cœur de la jungle citadine comme au creux des vallons laurentiens. Au-delà s'ouvraient des rues à maisons basses, s'enfonçant de chaque côté vers les quartiers de grande misère, en haut vers la rue Workman et la rue Saint-Antoine, et, en bas, contre le canal de Lachine où Saint-Henri tape les matelas, tisse le fil, la soie, le coton, pousse le métier, dévide les bobines, cependant que la terre tremble, que les trains dévalent, que la sirène éclate, que bateaux, hélices, rails et sifflets épellent autour de lui l'aventure. (44)

Nous retrouvons brièvement le quartier Saint-Henri transformé, déjà dépersonnalisé, à la fin de Les vivants, les morts et les autres. Mais le quartier montréalais où est située une grande partie de l'action de ce roman est Hochelaga, le quartier de la famille Lussier. Hochelaga, dans cette œuvre, est un univers humain essentiellement centré sur une institution dominante: la filature. La vie du quartier n'est que l'écho du drame qui

2. Tout au cours de cette étude, les chiffres entre parenthèses renvoient aux pages de l'œuvre dont il vient d'être question dans le texte. On trouvera, en appendice, la liste chronologique de tous les romans cités ou mentionnés. 
se noue, se déroule et se dénoue brutalement à l'usine entre le syndicat et la direction de l'usine. Dans Au pied de la Pente Douce et Les Plouffe au contraire, nous partageons encore, comme dans Saint-Henri, la vie unanime d'un quartier très défini de Québec, le quartier Saint-Sauveur. Ce quartier est un milieu social dans tous les sens du terme. Il possède son histoire, sa mentalité, son dynamisme propre, ses factions religieuses, ses clans politiques, sa hiérarchie officielle de chefs formels et ses hiérarchies officieuses de leaders naturels. Nous le voyons vivre, de façon picaresque, comme un univers communautaire sans la connaissance duquel les attitudes, les ambitions, les relations des personnages nous demeureraient étrangères. Au surplus, ce quartier des romans de Lemelin s'identifie avec une paroisse, Saint-Joseph. Milieu écologique et milieu religieux s'interpénètrent et sont absorbés l'un par l'autre. Nous y reviendrons.

L'histoire de plusieurs romans se déroule dans une petite ville. Rappelons, parmi les cas les plus attachants, la Fontile dans laquelle sont situés Ils posséderont la terre et Fontile de Robert Charbonneau - Fontile, « une drôle de ville, bâtie au milieu des marais» (Ils posséderont la terre, 83), à quelque deux cents milles au sud de Montréal. D'abord poste avancé d'une compagnie forestière, Fontile est devenue une ville industrielle de seconde importance $(10,13,14)$. Le grand-père du narrateur, Julien Pollender, en a fait son fief, un fief que contrôlent maintenant l'industrie et la banque (14). La génération du père de Julien « comptait plusieurs familles millionnaires... Leur éducation en faisait les égaux des familles de la capitale, mais retenus à Fontile par la volonté de leurs parents qui les liaient par testament, ils souffraient de leur entourage et le dénigraient» (14-15). Ces nouveaux riches ont ouvert un quartier neuf en aval de la ville et c'est surtout ce quartier que nous connaissons plutôt que leur " entourage» ouvrier qui habite les zones entourant les usines et le centre de la ville. «Vivre comme un homme de Fontile» (69) consistera, dans ce petit monde semi-bourgeois, «à nouer de solides relations, à se créer des habitudes: jouer aux cartes, boire un verre en compagnie, fréquenter quelques salons, peut-être un jour faire un beau mariage» (69). Dans le Saint-Joachim où habite pendant un temps Le libraire énigmatique de Gérard Bessette, nous faisons l'apprentissage, par les allusions discrètes qui la provoquent et les implacables conséquences qu'elle entraîne, de la force du qu'en-dira-t-on dans une petite ville. La vérité est ce que dit celui qui jouit auprès de ses concitoyens d'une excellente réputation (51). La norme qui retient d'agir selon son gré est que «ça ferait jaser les gens» (92). Quiconque est victime de la moindre réprobation a peur de se montrer dans la rue (131).

La petite ville qui s'incorpore le plus intensément aux attitudes et aux comportements de ses habitants est sans conteste le Macklin de Poussière sur la ville (Langevin). Cette petite ville minière affreusement laide (27) s'impose à nous physiquement et socialement, de jour et de nuit. Elle est plus que témoin de la tragédie qui s’insinue entre le docteur Alain Dubois et son épouse Madeleine. Elle s'y associe, la pénètre, l'accentue, l'oriente. Ce drame est vite devenu son drame. Au docteur Dubois qui sait déjà suffisamment que, dans une petite ville, «il en faut peu pour scandaliser» (65), le tout-puissant prêteur Arthur Prévost rappelle que «dans une ville comme Macklin, 
vous ne pouvez avoir de vie privée... Tout ce que vous faites, vous et votre femme, est fait devant toute la ville. Il est impossible de faire une carrière à Macklin si l'on n'a pas une conduite irréprochable. Le moindre faux pas est commenté et grossi démesurément» (132). La ville, qui sent en Madeleine « une de ses enfants» (138), prend parti pour celle-ci. Elle va forcer le docteur à quitter la ville (164); elle fait le vide autour de lui (172); resserre son étau (179-180). Une fois consommée la catastrophe de Madeleine, le vieux docteur Lafleur conseille à Dubois de partir: «... Vous êtes jeune. Vous pourrez recommencer ailleurs. Ici, il est trop tard» (208).

\section{LES FAMILLES}

Une puissance tenace, celle de la famille, guide l'action de la plupart de nos romans. Tantôt latente ou obscure, tantôt manifeste, toujours exigeante, cette puissance modèle le caractère des personnages, dispose les éléments de leur destin, détermine les conditions de leurs drames. En certains cas, la famille est elle-même le personnage central d'une histoire. Des romans sont des romans de la famille. Les exemples les plus ostensibles sont Bonheur d'occasion et Les Plouffe. Même dans les cas cependant où la famille n'occupe pas elle-même toute la scène, on en sent la sourde solidité. Les types de familles sont variés et correspondent aux milieux géographiques, aux classes ou aux segments de la société dont elles sont les microcosmes. Avec la campagne, la famille rurale est disparue de notre littérature. On l'évoque seulement pour expliquer l'ascendance des familles contemporaines ou comme objet de souvenirs. Elle appartient à une époque irrévocablement révolue. Les familles de nos romans sont des familles urbaines.

Ces familles urbaines, même une lecture rapide nous impose de les répartir en deux catégories nettement caractérisées. Il y a les familles des milieux ouvriers. Il y a les familles de type bourgeois ou de classe supérieure. Si l'on est très attentif, on peut discerner entre ces deux catégories un type intermédiaire de familles de "classe moyenne». Mais ces dernières ou les familles bourgeoises sont moins nombreuses que les familles ouvrières. Elles n'apparaissent qu'épisodiquement. Elles ne jouissent pas de la luxuriante abondance des descriptions consacrées aux familles de condition modeste, sauf dans Mon fils pourtant heureux de Jean Simard. Notons encore un trait qui semble révélateur. Les rares familles dans lesquelles le personnage important est le père sont de type bourgeois: le père est propriétaire d'une entreprise industrielle, homme d'affaires, membre d'une profession libérale, haut fonctionnaire ou politicien. À l'inverse, les familles où le personnage dominant est la mère sont en général de condition ouvrière. Un saisissant contraste entre ces deux types de familles est présenté dans une même œuvre, Les vivants, les morts et les autres de Pierre Gélinas. La famille de Maurice Tremblay qui habite l'avenue des Braves de Québec est dominée par le père, un industriel prospère; la famille des Lussier d'Hochelaga est régentée par une mère autoritaire et mesquine.

Voyons de plus près quelques-unes des familles patricentriques. Victor Debreux, l'ingénieur alcoolique dont Thériault nous raconte la déchéance dans Cul-de-sac, a eu un père "très digne et... empesé» (98). Il a été lui-même un enfant modèle (51). Il a 
eu au-dessus de lui, non seulement ce père mais un oncle, des oncles, et encore audessus un grand-père: tout un aréopage de mâles qui ont décrété pour lui l'orientation et les normes de son existence. C'est un oncle qui a décidé le choix de sa carrière. Le bureau d'ingénieurs dont il devient un associé subalterne lui fait penser à une famille, c'est-à-dire un système dans lequel le commandement est mandaté à des porte-parole nettement définis. C'est son père qui, après une première phase de sa déchéance, lui propose la forme salvatrice d'une existence nouvelle. Dans Fontile, la structure familiale qui entoure Julien Pollender est durcie au sommet et laisse une sorte de vide immédiat autour du héros. Ici encore c'est un grand-père qui est chef — «chef de la tribu» (19) - et le père de Julien est entièrement soumis à ce grand-père; il ne s'occupe pas de Julien (48). Celui-ci, sa mère étant morte lorsqu'il avait trois ans, est élevé entre une belle-mère et une tante, «dans la jupe des femmes » (35), comme il le rapporte avec une cruelle précision. Du père Victor Tremblay, dans Les vivants, les morts et les autres, nous savons qu'il jouit auprès de ses trois fils du même écrasant prestige que dans l'ensemble de la société $(24,27,30)$. Un de ces fils, Lucien, a fui le foyer paternel. Maurice fera bientôt de même. Le père, d'ailleurs, meurt dès le début du roman. Le titre du roman de Jean Simard, Mon fils pourtant heureux, laisse par lui-même entrevoir l'incompréhension qui caractérisera les relations entre Fabrice et son père - un père modérément voltairien, hautain, solitaire. Fabrice vit «entre une mère effacée et un père morose» (72). Chaque dimanche, les trois se retrouvent pour un sombre repas de famille chez une grand-mère maternelle qui se fait auprès de ses filles mariées l'intransigeante prédicatrice d'une aveugle soumission des femmes à leurs maris. Le père de Fabrice meurt stoïquement après une longue agonie subie avec bravade.

Dans aucun de ces romans, il n'y a d'affrontement viril entre fils et père. Ou le père meurt très tôt, ou il est pratiquement inexistant, entièrement subjugué lui-même par la présence de son propre père. Le «meurtre du père» n'a jamais lieu dans notre littérature - sauf dans un seul cas qui est une exception: celui du Denis Boucher d'Au pied de la Pente Douce. Non seulement Denis a fait chanter son père (191) mais, dans une scène à laquelle je ne trouve d'équivalent dans aucun autre roman, il s'oppose radicalement à lui pour affirmer ses prérogatives d'homme (269). Le même Denis déjà avait proféré ce mot annonciateur du programme de toute une vie autonome: «Pauvre Jos, ton fils fera ce que tu as raté. J'écrirai, moi» (153).

Trois familles au moins s'imposent comme exemples de familles matricentriques: la famille Lacasse de Bonheur d'occasion, Les Plouffe, la famille Lussier dans Les vivants, les morts et les autres. Les trois, nous l'avons noté, sont des familles ouvrières. Dans chacune, non seulement la mère est le personnage central mais elle est toute la famille. Elle est le carrefour des rares contacts et de la communication évasive entre les membres de la famille. Elle contrôle leurs rapports et leurs statuts respectifs. Elle est celle qui retient en quelque sorte en elle l'existence que ses enfants lui ont prise. Relevons quelques traits révélateurs de ces trois univers familiaux.

L'existence de la famille Lacasse baigne dans la grisaille et les bruits ferroviaires du quartier Saint-Henri. Chaque jour qui commence aura un crépuscule hypothétique. Il 
y a eu le chômage et il y a la guerre qui commence. Il n'y a jamais d'argent. L'angoissante insécurité croît au rythme de l'inconstance d'Azarius, le père velléitaire. C'est une existence morne, sans espoir. Je trouve un symbole de l'ennui indéfini de ses travaux et de ses jours dans le ronronnement de la machine à coudre de Rose-Anna:

La roue de la machine se reprit à tourner; elle tournait entre l'ennui de Florentine et la rêverie décousue de Rose-Anna, elle tournait comme les années avaient tourné, comme la terre tournait, ignorant dans son cycle éperdu ce qui se passe d'un pôle à l'autre. Ainsi la maison semblait prise dans ce mouvement inlassable de la roue. La besogne emplissait la maison; elle rejetait la parole, toute compréhension. Elle filait, les heures avec elles, les confidences perdues avec elles, et tant de voix se taisaient, tant de choses restaient inexprimées pendant qu'elle ronronnait, elle, l'infatigable. (227)

C'est Rose-Anna qui coud et c'est elle qui fait tourner la vie de la famille. Chaque année, il faut déménager: c'est elle qui part à la recherche d'un nouveau logis $(89,91$, 95). Pourtant, se dit-elle et nous dit l'auteur, «elle n'était pas née pour mener» (223). Elle ne demanderait qu'à croire son mari et à l'écouter. Mais elle ne peut "prendre appui sur aucun des siens» (223). Elle est aveuglement bonne, spontanément poussée à donner, résignée devant la vie, devant les siens. Elle est résignée au delà de toute épreuve et de tout sentiment. Que peut-elle avoir hérité d'autre d'une mère fataliste «qui avait parlé toute sa vie de résignation chrétienne et de douleurs à endurer» (226) et qui, lors de la fameuse visite de ce dimanche de printemps, après plus de vingt ans de séparation, ne trouve rien d'autre à lui dire que:

Pauv' Rose-Anna, j'ai ben pensé que t'avais eu de la misère, toi aussi. Je le savais ben, va. Ça pouvait pas être plus drôle pour toi que pour les autres. Tu vois astheur que la vie, ma fille, on arrange pas ça comme on veut. Dans le temps, tu pensais avoir ton mot à dire... toi... (264)

Rose-Anna voudrait empêcher Florentine d'être malheureuse, Eugène de s'enrôler, le petit Daniel de mourir. Mais elle n'a pas le temps de jongler. Pour elle seule, la vingtaine de logis que la famille a successivement habités dans Saint-Henri se télescopent dans le mot «chez-nous» — un mot d'une « richesse sourde, cachée, infinie qui n'a de sens que pour Rose-Anna» (379-380).

On est encore ensemble, dit-elle à Azarius, après le dernier déménagement. On a encore not' force, not' santé. Qu'est-ce que tu veux qui nous arrive de pire? C'est encore avec nos paires de bras qu'on se tirera d'affaire, va, crois-moi. Des jongleries, c'est pas ça qui aide. Des jongleries! (389)

Le grand «jongleur», bien sûr, c'est Azarius (120). Une fois tombée sa jactance et passées ses fanfaronnades, il redevient l'homme incertain, contrit, sans ressort:

Un homme s'asseyait auprès du poêle de cuisine et s'étirait paresseusement. Je crois ben qu'on va se laisser vivre, sa mère, en attendant... Tant qu'à pas travailler de mon métier !... (214)

Il est serviable jusqu'à l'abdication de tout amour-propre. Temporairement sans emploi, il s'offre aux travaux du ménage: «Va te recoucher sa mère; je ferai ton bardas 
aujourd'hui» (221). À la fin de l'histoire, d'un coup de tête, Azarius aura pris une invraisemblable décision. Devant une Rose-Anna qui hurle de stupéfaction, il apparaît revêtu de l'uniforme des conscrits. Il s'est enrôlé dans l'armée. «... le plus beau de toute, finit-il par avouer à Rose-Anna, c'est que tu vas être débarrassée de moi» (515). Rose-Anna se souvient-elle sans surprise, à ce moment-là, de la dernière phrase murmurée, sur son lit de mort, par l'homme qui a été son propre père: «Enfin, tu vas être délivrée d'une de tes croix, ma pauv' femme!» (266)?

La maison des Plouffe de Lemelin, c'est avant tout une cuisine. La reine de ce domaine, c'est madame Plouffe:

Cette vieille femme, aux chairs amples, flasques et ridées, qu'un foie malade teintait de jaune, qu'une trop tardive maternité avait épuisée, se gonflait soudain sous le coup d'une impérieuse volonté comme une outre vide depuis des années qu'on remplit soudain d'eau fraîche. Avec un fatalisme aveugle qui n'était même pas de la résignation, madame Plouffe avait subi ses épreuves sans murmurer, d'un air à peine ennuyé: ses fausses couches, son mari ivrogne, ses enfants capricieux. Depuis quarante ans elle allait et venait dans sa cuisine, préparait les repas, lavait la vaisselle, mangeait les restes de nourriture que les enfants n'aimaient pas. (83)

Son mari Théophile est un simple acolyte dans la liturgie de discussions et de tensions qui règle les rapports entre les quatre membres de la famille: Napoléon, l'aîné puéril et sympathique; Cécile, célibataire par la faute de la mère; Ovide, l'intellectuel; Guillaume, le sportif. C'est madame Plouffe qui, dans cette famille d'adultes, prend toutes les décisions, avec l'aide et la collaboration, s'il le faut, du curé (86). Les enfants ont cependant des sursauts d'indépendance et de colère. Ainsi Napoléon, le jour où il décide que le pasteur protestant Brown reviendra à la maison, malgré la défense de sa mère, lui porter des photographies sportives. Une telle décision «foudroyante» enchante le père qui sourit «de contentement de se voir vengé du despotisme de cuisine de sa femme par l'aîné de ses fils» (87). Par contre, cette décision déclenche comme une balle une riposte mélodramatique d'Ovide: «Tu n'as pas honte de faire pleurer ta vieille mère de soixante ans?» (88). Chacun, tour à tour, cherche à s'affirmer mais, chaque fois, s'attire une réprimande de l'un des autres qui assume le rôle maternel. Ovide, un jour qu'il a bu — les hommes dans les romans canadiens ne parviennent en général à s'exprimer complètement que lorsqu'ils sont ivres ou à demi ivres — lâche le grand mot: «Oui, on est forcé d'être des enfants toute sa vie. C'est pour ça que ceux qui veulent devenir des hommes sont malheureux...» (146). Cécile, de son côté, accuse sa mère d'égoïsme parce que celle-ci l'a empêchée de se marier quand et avec qui elle le voulait. «J'étais votre seule fille. Vous me disiez que le mariage apportait seulement la misère. Donc, je me suis pas mariée. Maintenant vous m’enlevez mon seul ami. Égoïste! Sans-cour !...» (159).

La mère, à la longue, ne peut réprimer son dépit. Devant Napoléon qui lui demande timidement s'il peut amener une «blonde» à la maison, elle éclate rageusement: «C'est ça... Mariez-vous donc tous! Abandonnez vos vieux parents. Vous ne leur reprocherez pas de vous avoir gardés pour les désennuyer!» (160). Et ainsi va 
l'existence des Plouffe, de protestation en apaisement. La mère Plouffe voit son domaine «lui (être) enlevé par tranches à mesure que les enfants (vieillissent)» (83), mais elle persiste à proclamer que «c'est elle qui mène» (264). Elle ne souffre pas que son autorité soit partagée. Elle tolère à peine la présence physique de son mari. Celui-ci devenu gréviste ne quitte plus la maison. «La catastrophe... c'était l'énorme présence de Théophile dans la maison durant la journée» (214). Comme Azarius Lacasse, Théophile Plouffe offre piteusement son aide: «Où sont les torchons?...», demandet-il à madame Plouffe (214). Celle-ci le rabroue. Théophile, mû par un sentiment semblable encore à celui d'Azarius, laisse échapper: "Vous m'enduriez mieux dans le temps que je travaillais. Si je peux mourir, vous allez être débarrassés» (214).

Nous pénétrons chez la famille Lussier, dans Les vivants, les morts et les autres, au moment où la mère, Rachel, vient de recevoir un télégramme lui apprenant que l'un de ses fils, Pit, soldat de l'armée canadienne, a été tué en Corée. Cet événement précipite la déchéance de la veuve de Pit, Yolande, qui n'a jamais été qu'une étrangère dans la maison. Son deuil même lui est enlevé. Le clan des Lussier se resserre et l'écarte: «Quand elle (Yolande) retrouva les sens, la mort de Pit, la douleur et le deuil lui avaient été arrachés à elle, sa femme ; la famille Lussier les avait réquisitionnés et monopolisés » (42). Ce conflit belle-mère-bru ne fera que s'accentuer par les insinuations, la calomnie et le chantage, si bien que Yolande, moralement exténuée, finira par quitter cette maison et s'abandonner graduellement à un destin dégradant. La mère Lussier demeure maîtresse du clan mais elle assiste, comme madame Plouffe, à «l'effritement de son domaine» (45). Elle est d'origine rurale, comme son mari, Wilfrid, qui au moment de leur mariage est venu chercher du travail à Montréal. C'est Montréal qui a ébranlé la structure de la vie familiale telle qu'elle l'avait connue dans sa jeunesse, alors que «la mère régnait sur un foyer paisible dans un pays sans histoires» (45). Le chômage des années de crise a retenu son mari à la maison. La guerre l'a renvoyé à l'usine. Oscillations entre le spectre du chômage en temps de paix et les brèves euphories en temps de guerre. «Rachel était emprisonnée dans un cercle implacable...» (46). Nous assistons ainsi au règne incertain d'une mère désenchantée, volontiers acariâtre et dont l'animosité s'exerce vivement, une fois sa bru disparue, contre sa propre fille Réjeanne, syndicaliste militante. Par celle-ci, un conflit du vaste univers ouvrier est introduit dans l'univers Lussier où il provoque une large fissure. Dans ce conflit, le père, ouvrier à Canadair, prend le parti de la soumission. De même qu'il se courbe sous les impératifs de sa femme, il préconise la docilité aux patrons. Sa philosophie fataliste est toute voisine de l'attitude du campagnard devant les forces de la nature: «Les compagnies sont plus fortes que vous autres. Vous parlez pour rien» (50). Le pauvre Wilfrid connaîtra une lamentable fin: il retombe en enfance. Rachel devient une sorte de veuve (291). Elle s'institue gérante de la maigre fortune d'une belle-sœur, Maria, dont le mari vient de mourir. Les deux veuves vont «s'établir» en achetant une maison dont elles occuperont un étage et loueront les autres. Wilfrid est pratiquement remisé dans un placard (292). C'est le règne des veuves.

Cette esquisse de deux types de familles, bourgeoises et patricentriques, ouvrières et matricentriques, est loin d'épuiser la richesse de l'«être familial» dans nos romans. 
Elle suggère tout au plus un fond de scène. Elle révèle aussi un schéma général: l'aliénation du père et l'omnipotence de la mère. Mais ce schéma comporte des variations. Il y a des contre-parties de la mère des milieux ouvriers dans les milieux bourgeois: il y a la mère surprotectrice et despotique d'Edward dans Ils posséderont la terre de Robert Charbonneau; il y a la mère couveuse d'Yvon Letellier dans Pierre le magnifique; il y a la mère étrangère de Pierre Boisjoly lui-même. Et il faudrait encore raffiner cette typologie pour en cerner les modalités labyrinthiques, par exemple dans les drames œdipiens de Jean Filiatrault. Aussi bien, une sociologie plus complète de notre littérature devrait s'interroger sur le fait qu'un très grand nombre des familles dans les romans sont des familles incomplètes. Et ce phénomène n'est pas récent: il date des tout débuts de notre littérature. Ainsi, dans trois de nos romans du Xıx ${ }^{\mathrm{e}}$ siècle, Charles Guérin de P.-J.-O. Chauveau, Jean Rivard de Gérin-Lajoie et Robert Lozé d'Errol Bouchette, il est fait mention de cinq familles. Or, aucune de celles-ci n'est complète. Il y manque invariablement soit le père, soit la mère. Il en est encore fréquemment ainsi dans nos romans contemporains. Il faudrait expliquer cette constante. N'est-ce pas d'elle, comme des quelques schémas fondamentaux des relations père-mère-enfants que nous venons de noter, que dérivent les attitudes d'impuissance, d'insécurité, ou de révolte devant l'existence qui caractérisent les Jean Cherteffe, les magnanimes mesquins d'André Giroux, les intellectuels angoissés de Robert Charbonneau et de Robert Élie, les révoltés de Claude Jasmin? Si l'on veut entreprendre une psycho-sociologie ou une psychanalyse de ces personnages, il faudra scruter plus à fond ces manques de plénitude, de transcendance, de conditionnements positifs dans l' «être familial» de nos romans.

\section{INSTITUTIONS DOMINANTES}

Arrêtons-nous à certaines institutions qui, en tant que postes d'exercice du pouvoir, dominent la vie collective.

Il faut revenir à la paroisse que nous avons déjà signalée comme cadre géographique. Elle est aussi une structure religieuse et sociale. En tant que telle, les romans récents nous la présentent de l'extérieur plutôt que de l'intérieur. Elle est un ensemble d'activités sociales dont les mécanismes sont animés sinon démontés sous nos yeux, souvent pour notre amusement. C'est à une opération de ce genre que se livre Thériault sur la paroisse de Saint-Léonide-le-Confesseur dans Les vendeurs du temple. Il en est de même pour la paroisse Saint-Joseph dans laquelle se déroulent les deux premiers romans de Lemelin. La paroisse Saint-Joseph est la «famille» du curé Folbèche:

Sa paroisse! C'était une famille de plusieurs milliers d'enfants, à la mesure de son rêve de prêtre, et dont il avait pris charge vingt-cinq ans auparavant. Il lui semblait les avoir adoptés et tenus tous au berceau, même les vieillards. Et il les avait élevés avec la poigne solide d'un vrai père, leur appliquant du haut de la chaire de magistrales fessées et au besoin leur racontant des histoires de croquemitaines pour venir à bout de leurs caprices de gamins, ou pour les punir de n'avoir pas obéi à leur mère la Sainte Église. Lentement, la famille s'était formée, unie. L'enfance de sa paroisse, si elle lui avait fait passer des nuits 
blanches, avait aussi été bien douce à son amour paternel. Cette durable enfance n'avait pas, à son avis, empêché la famille de prospérer, de se bâtir une belle église, un beau presbytère, de fières écoles. Mais la dangereuse et ingrate période de l'adolescence et de la jeunesse est toujours à craindre. Comment l'empêcher de surgir! La famille ne se rebiffaitelle pas aujourd'hui que le père voulait se reposer? Elle le traitait de vieux démodé et prétendait user de la formation et de l'esprit catholiques pour se conduire elle-même. Elle avait lu les journaux, interprétait à sa façon la guerre d'Espagne et discutait les sermons, critiquait les prélevés sur les revenus que le père exigeait d'elle. Jusqu'aux marguilliers qui voulaient prendre des décisions! (68-69)

Cette famille paroissiale tient surtout son unité d'un attachement routinier à des pratiques conventionnelles de piété et à une soumission, d'ailleurs de plus en plus contestée, à l'autorité du curé. La préoccupation principale de celui-ci est la réussite du projet de construction d'une nouvelle église, le succès de ses mercredis à Saint-Joseph, l'organisation et la surveillance des «bingos» périodiques. Le grand souci de sa pastorale est de conjurer tout courant de pensée qui contrarie les normes de son moralisme simpliste en le stigmatisant par l'épithète de «communiste». Tour à tour tyrannique et opportuniste, il devient un docile instrument dans la main d'un astucieux Denis Boucher pour peu que celui-ci lui prouve que sa présence à un événement sportif est de nature à rehausser son prestige aux yeux de ses paroissiens. Dans un moment de crise ou de danger menaçant l'un ou de l'autre de ceux-ci, il surgira comme une mère protégeant sa couvée: les policiers de la gendarmerie royale vont arrêter Guillaume Plouffe qui a lancé une balle à travers le cortège royal qui passe par les rues de SaintJoseph; le curé leur défend d'y toucher et le réclame comme sien: «It is my business. It is my parish, you know. Him a good boy. I order you to give liberty to Guillaume. Understand?» (Les Plouffe, 185).

Il est plus malaisé de déterminer la fonction que joue l'armée et la signification que revêt la guerre dans la vie des personnages de romans. L'armée apparaît dans ceux-ci avant l'usine. Elle est ce à quoi s'opposent le plus violemment les impulsions d'auto-affirmation et elle est aussi l'occasion inespérée, indiciblement bienfaisante, d'un emploi assuré, d'un salaire, d'une sécurité, à la suite des désespérances des années de crise économique. Telle elle se présente aux jeunes chômeurs de Bonheur d'occasion. Pour un Guillaume Plouffe, le sportif, elle sera le cadre d'un sport suprême. Le cri final de mère Plouffe à la fois terrifiée et exultante: «C'est pas croyable! Guillaume qui tue des hommes!» n'est guère différent de celui par lequel elle eût annoncé à tout le quartier que Guillaume était devenu le champion lanceur des États-Unis. Pour d'autres, comme pour Fabrice Navarin, l'armée, en tant que structure autoritaire, représente la forme extrême, implacable, de ce qui est le plus honni dans la vie sociale. "Je ne pouvais, dit Fabrice, manquer d'apercevoir entre la famille, l'Église, l'école et l'armée, je ne sais quelle analogie, quelle honteuse connivence, quelle identité de méthodes proprement intolérable; et consistant, dans la plupart des cas, à briser l'être afin de mieux l'asservir...» (Mon fils pourtant heureux, 173). Pour d'autres, comme pour le frère de Jean Cherteffe d'Évadé de la nuit, Marcel, l'armée aura été une occasion de s'affirmer d'une façon violente, frustratoire, au-delà des angoisses spirituelles et des sentiments 
les plus profondément humains. De s'affirmer aussi en tant que force dans l'action. Elle lui aura procuré «la satisfaction de l'homme d'action qui découvre un événement à la mesure de ses désirs, la fierté de commander des hommes forts comme lui, ne laissant derrière eux aucune attache qui eût pu gêner leur aventure » (Évadé de la nuit, 150). Deux romans, Neufjours de haine de Jean-Jules Richard (1948) et Les Canadiens errants de Jean Vaillancourt (1954) sont exclusivement consacrés à l'analyse explosive de la guerre génératrice d'héroïsmes, de paradoxes, d'horreurs, d'absurdités et de contresens qui remettent mystérieusement en cause les valeurs humaines élémentaires. Une fois la guerre terminée, les héros de romans qui y ont participé reviennent à la vie civile entourés d'un halo de prestige vite oublié. Ce prestige leur vaut cependant une ascension automatique de quelques places dans les rangs de la société, particulièrement dans leurs occupations de comptables, de fonctionnaires, d'ingénieurs, de prêtres, d'intellectuels...

L'usine, avons-nous dit, apparaît tardivement dans le roman. Depuis longtemps pourtant celui-ci a parlé d'ouvriers et de gagne-petit. Il y a des ouvriers dans Fontile. Des ouvriers vont et viennent dans Bonheur d'occasion, dans Poussière sur la ville. On sait qu'ils sont typographes, mineurs, bûcherons, tisserands. Mais rarement les voit-on dans le lieu même de leur travail. Avant de connaître celui-ci, nous ferons d'abord l'expérience, dans nos romans, de la grève. Les grèves ont été nombreuses dans les romans des dernières années. Lemelin décrit une grève des typographes au journal L'Action chrétienne dans Les Plouffe et une grève de bûcherons dans Pierre le magnifique. Vaillancourt a consacré à une grève célèbre un livre entier, Le feu dans l'amiante, qui a des moments d'une sauvage et spectaculaire beauté qu'on dirait détachés d'un film d'Eisenstein. Gélinas, dans Les vivants, les morts et les autres, décrit trois grèves différentes: une dans un camp de bûcherons, une autre chez les tisserands de la filature d'Hochelaga, une grève au magasin Dupuis, sans compter l' «émeute Richard» de 1955 au Forum de Montréal! La grève du syndicat des tisserands occupe cependant dans ce roman une place prédominante. Les péripéties en sont racontées avec une précision technique, depuis les signes avant-coureurs dans les rapports entre contremaîtres et ouvriers, les tumultueuses assemblées des syndiqués, les longues soirées passées à discuter de stratégie, jusqu'aux jours pénibles de la mise à pied, à l'inlassable piquetage, au pseudo-règlement, par-dessus la tête du syndicat, entre la compagnie et le directeur américain d'une centrale syndicale rivale.

C'est dans ce même roman qu'est évoquée pour la première fois, à ma connaissance, la vie interne d'une usine. Une belle page décrit les étapes de la marche du fil à travers les secteurs de la filature:

Le fil (que Réjeanne) manipulait sur sa machine avait été traité d'abord dans le département du père Dorval, dont les trois filles et les deux garçons travaillaient aussi au moulin; il passait ensuite dans le département de la vieille Lanctôt, Lucie, Ti-Coune Bédard et le «rat à Germain»... Le fil était entre eux le lien tangible. Les opérations se répartissaient sur cinq étages et sur une longueur de deux pâtés de maisons, mais le travail de chacun était le maillon nécessaire de la chaîne unique qui était à la fois l'image de leur dépendance individuelle et de leur force collective. (Les vivants, les morts et les autres, 36) 
Un autre passage du même roman décrit l'ahurissement et l'abrutissement d'un apprenti dans un atelier d'embouteillage:

C'était la première fois de sa vie qu'il pénétrait dans une manufacture. On l'avait placé devant une chaîne sur laquelle roulait interminablement une procession de bouteilles propres à donner le vertige. Gérard imprimait à la machine le petit rectangle rouge sur la bouteille d'eau gazeuse «7 Up», les deux bras enduits de peinture jusqu'au coude. À cela, il se serait acclimaté; il n'avait pu s'accorder à la cadence du roulement. Un relâchement, une minute de distraction, et les bouteilles le dépassaient; impossibles à rattraper, elles allaient se fracasser plus loin dans un vacarme assourdi par le beuglement du contremaître. Habitué au travail individuel des chantiers, fier de sa capacité d'abattre entre l'aurore et le crépuscule quatre cordes dans le beau bois, ces huit heures de travail insignifiant, que des femmes accomplissaient sur la même machine plus rapidement et mieux que lui, l'humiliaient et l'abrutissaient. Il s'en était pris à lui-même d'être plus épuisé par une petite journée à la Consumers Glass que par douze heures dans les chantiers de Murdoch. Un jour, il était revenu chez son oncle Wilfrid en expliquant qu'il avait été congédié. Il n'avait pas travaillé depuis... (147-148)

Un des caprices les plus singuliers de notre littérature est le peu de cas qu'elle fait du collège. Cette institution pourtant cruciale dans la vie canadienne-française est presque passée sous silence. Nous n'avons pas encore de roman de la vie collégiale. On fait allusion au collège dans Fontile et dans plusieurs des romans psychologiques à cadres bourgeois. De temps à autre, un personnage va consulter un ancien professeur ou un ancien directeur spirituel dont il a besoin en un moment de déboire ou de crise. Gilles Marcotte, il est vrai, évoque le grand séminaire comme une réalité qui fait partie de la substance humaine et spirituelle de son douloureux abbé Savoie. Lemelin, au début de Pierre le magnifique, décrit une cérémonie de distribution de prix au petit séminaire de Québec mais nous ne connaissons pas la vie de l'institution, même si Pierre Boisjoly rend, une fois ou deux, visite à son étrange ami l'abbé Lippé. Seul Jean Simard nous fait accompagner le Fabrice de Mon fils pourtant heureux dans cette geôle pour adolescents dont la vie grégaire lui paraît encore moins tolérable que la vie de famille. Fabrice qui est un «fils à maman» (113) envie sans pouvoir y prendre part la «brutale familiarité» qui unit les camarades de collège (117). Les quelques années d'internat au séminaire seront pour lui les plus beaux jours d'une nouvelle mort.

Une institution, par contre, qui est présente de façon presque surprenante dans nos romans est l'hôpital, ou sa version spécialisée, le sanatorium. Nombreuses sont les œuvres dont des épisodes importants, quelquefois décisifs, se passent à l'hôpital. Celui-ci est tantôt un lieu d'appréhension, tantôt le lieu de la réalisation d'un désir inavoué d'abdication et de repos indéfinis. Dans l'esprit des pauvres gens, comme le rappelle le docteur Lafleur au jeune docteur Dubois de Poussière sur la ville, l'hôpital est «le vestibule du cimetière» (114). Il y a l'hôpital où meurt le petit Daniel Lacasse de Bonheur d'occasion; l'hôpital où, dans Évadé de la nuit, se rencontrent Jean Cherteffe et Micheline Giraud au chevet du fils mourant de Roger Benoît; l'hôpital d'Alexandre Chenevert; l'hôpital de Jean Sirois dans Le gouffre a toujours soif de Giroux; l'hôpital où, dans Le poids de Dieu, l'abbé Savoie bénit les étranges fiançailles 
de Serge Normand et de Marie Norbert; l'hôpital où le courageux Napoléon Plouffe conduit sa nouvelle amie qui, contre toute attente et peut-être seulement par la magie de l'amour de Napoléon, va renaître à la vie... L'analyse du symbolisme de l'hôpital, comme l'analyse de la famille et peut-être pour des raisons identiques, devrait tenter les psychologues.

\section{TYPES PROFESSIONNELS}

Quels sont, à l'intérieur ou à la périphérie de ces structures institutionnelles, les types professionnels qui se dégagent avec le plus de relief de notre comédie humaine romanesque?

Il est impossible de ne pas nous arrêter d'abord au prêtre. Gilles Marcotte, avec Le poids de Dieu, a doté notre littérature de son premier roman authentiquement sacerdotal. Pour la première fois, un homme consacré à Dieu, l'abbé Claude Savoie, cherche à comprendre le sens de son oblation et à assumer les bouleversantes exigences d'un don réel de soi aux hommes. Déjà, dans Le temps des hommes, Langevin avait créé un personnage de prêtre, Dupras, torturé par le désir d'aller aux hommes en assumant l'univers des hommes. Mais ces œuvres sont des exceptions. Quelques impressionnantes figures de prêtres cependant apparaissent discrètement avec grande miséricorde et grande douceur au chevet d'un Jean Sirois ou d'un Alexandre Chenevert mourants. En général, les prêtres qui circulent dans nos romans sont surtout porteurs d'un statut social et nantis de privilèges de toutes sortes. Ils sont respectés ou raillés, écoutés ou contredits, selon qu'on accepte ou qu'on rejette les formes traditionnelles d'autorité et le style ecclésiastique de contrôle social dont ils sont les véhicules. Tels sont le curé Folbèche (Au pied de la Pente Douce, Les Plouffe), le curé Loupret (Pierre le magnifique), le curé Bossé (Les vendeurs du temple). Tels sont la plupart des prêtres professeurs, aumôniers, directeurs, auxquels sont associés de gré ou de force les personnages de romans.

À un niveau voisin de celui du prêtre se situe le frère enseignant qui, pour la première fois aussi, a fait son entrée dans notre littérature avec Les élus que vous êtes de Clément Lockquell - le «frère» cet oublié, que Lockquell a dépeint avec une si courageuse lucidité, et dont on comprend à demi-mot que son plus grand renoncement est de ne pouvoir partager, lui qui pratique des vertus identiques à celles du prêtre, un statut social identique à celui que la société reconnaît au prêtre...

Poursuivons l'examen de cette galerie de types professionnels en tâchant de suivre d'aussi près que possible l'ordre de prestige dont jouissent leurs titulaires dans leur existence romanesque. Il y a dans nos romans quelques juges. J'en choisis deux au hasard: le père d'Edward Patry dans Les vivants, les morts et les autres, et le juge Giraud, le père de Micheline dans Évadé de la nuit. L'un et l'autre se pensent et s'affichent comme des incarnations de l'ordre social, des instruments de la moralité publique, des parangons de vertu. Livrés à eux-mêmes, il leur arrive de se poser avec un certain frisson quelques-unes des questions dont ils cherchent professionnellement les réponses chez les accusés traînés devant eux. Les premiers romans canadiens du 
$\mathrm{XIX}^{\mathrm{e}}$ siècle accordaient une place de choix à l'avocat. Celui-ci, sans avoir tout à fait disparu de la littérature, ou bien est éclipsé par les protagonistes d'une société devenue industrielle, ou bien disparaît sous les traits de l'homme politique, du journaliste, du fonctionnaire. On continue, par contre, d'entourer les médecins d'une vénération ambiguë qui englobe tout à la fois le respect superstitieux, la crainte et, en définitive, la confiance dans un pouvoir magique.

Quant aux hommes d'affaires et aux chefs d'entreprise, ils sont de plusieurs catégories et de prestige variable. Un Marius Tremblay (Les vivants, les morts et les autres), grâce à son audace et à des contrats de l'armée durant la guerre, a fait d'une boutique de mercerie une florissante entreprise. Son fils Victor héritera "plus que d'une fortune... d'une tradition, d'un nom, d'une responsabilité» (27). Un Willie Savard, dans Pierre le magnifique, est propriétaire d'une exploitation forestière qu'il dirige avec la faconde d'un directeur de cirque.

Aussi variés par l'occupation et le tempérament sont les fonctionnaires. Jean Simard a campé un portrait théâtral du haut fonctionnaire qu'est le père de Fabrice Navarin. Dès sa sortie de l'université, il est devenu

secrétaire d'un ministère; puis, sous-chef intérimaire d'un département; et ainsi, d'intérim en intérim, il gravit patiemment les degrés du fonctionnarisme, pour se fixer en définitive à un niveau élevé, proche du sommet — mais non point sur un pic vertigineux. Un haut plateau, de préférence, convenant parfaitement à son caractère ambivalent: à la fois couard et autoritaire. Car, lorsqu'on craint les hommes, autant devenir leur chef. Par ailleurs, il est rassurant de dépendre soi-même d'une autorité impavide, capable d'assumer la responsabilité des décisions les plus écrasantes... (54)

Une faune de moyens et petits fonctionnaires s'agitent dans les romans d'André Giroux: monsieur Laberge, Édouard Giguère dans Au delà des visages; Jean Sirois dans Le gouffre a toujours soif. Et comment oublier le saisissant Alexandre Chenevert de Gabrielle Roy? Alexandre Chenevert, le caissier anonyme de la Banque d'Économie — le «petit homme» de la grande ville, isolé de tous et de lui-même, une sorte de Léopold Bloom à rabais, qui reste à son poste «comme restent dans leur vie presque tous les humains» (43).

Je mentionnerai, pour clore cette liste incomplète, le personnage de l'écrivain qui revient dans les romans avec une persistance égale à l'uniformité des traits sous lesquels il se présente. L'écrivain, c'est Julien Pollender (Fontile), c'est Denis Boucher ( $A u$ pied de la Pente Douce), c'est Fabrice Navarin (Mon fils pourtant heureux), c'est le Gérald Forrestier du Prix David d'Émile-Charles Hamel. L'écrivain, c'est l'intellectuel sous toutes ses formes mais particulièrement celles du jeune étudiant, du journaliste, du fonctionnaire. L'écrivain des romans, dont les affirmations souvent résonnent comme celles du «je» à peine travesti du romancier lui-même, incarne l'ambition la plus résolue d'émancipation sociale dont nous pourrons évaluer plus exactement la signification si nous la situons dans le contexte des classes et des structures globales de la société. 


\section{LES CLASSES SOCIALES}

Qu'il existe des univers sociaux différenciés, les distinctions entre les quartiers bourgeois et les quartiers ouvriers des grandes et petites villes, entre les types de familles, entre les catégories professionnelles à l'intérieur desquels se meuvent les personnages de romans, le manifestent abondamment.

Cette démarcation entre strates sociales se décèle à des indices subtils ou massifs par lesquels les individus ou les groupes signalent les distances psychologiques, intellectuelles ou morales qui les séparent. Les exemples de ces distances foisonnent et nous n'avons que l'embarras du choix. Dans Fontile, Georges Lescaut, qui vient d'être mis en apprentissage, impose à son ami Pollender «de l'abandonner à son nouveau milieu de petits employés» (44). Dans Au pied de la Pente Douce, Gus Perreault, le petit fonctionnaire organisateur politique, est roi au milieu des ouvriers qui ont la hantise d'un emploi stable (34). Florentine Lacasse (Bonheur d'occasion) est mal à l'aise, malgré son émoi amoureux, de se rendre à la réception d'Emmanuel Létourneau qui habite la place Sir-Georges-Étienne-Cartier. Puisque sa mère a déjà été femme de peine chez les Létourneau, est-il étonnant que monsieur Létourneau déplore que son garçon fait une folie... qu'il «ne tiendra jamais son rang» (179)? Et que dire du mélange de rêverie admirative, de silencieuse hostilité que provoquent chez les Lacasse, chez Emmanuel lui-même, les quartiers des millionnaires anglais des pentes du Mont-Royal? Le petit Daniel Lacasse qui est transporté, avant de mourir, dans un hôpital de ce quartier, habite dorénavant, même avant sa mort, un univers doublement étranger, au delà de tout espoir des habitants de Saint-Henri, représentant déjà presque la béatitude... C’est à la fois une différence d'altitude géographique et une différence d'altitude sociale qui éloignent de la «Haute-Ville» de Québec la famille Boucher, la famille Plouffe, toutes les familles des romans de Lemelin. La dichotomie Haute-Ville - Basse-Ville tronçonne la société québécoise en deux mondes dont chacun a sa mentalité, ses canons sociaux, ses organisations politiques, ses clubs sportifs.

Aussi bien, certains univers sociaux ont des attributs, des comportements caractéristiques. La vie familiale en milieu ouvrier se déroule dans une seule pièce de la maison - la cuisine; ou encore dans la cour. Le lieu de rencontre des jeunes est le restaurant du coin et celui des hommes, la taverne. Les discussions conjugales, les conversations ou les visites entre amis des milieux bourgeois prennent place, elles, dans les salons et les boudoirs. Le lieu de rencontre des hommes est le club et celui des jeunes, le bar chic. Être de «bonne classe», c'est-à-dire de classe élevée, signifie posséder de «bonnes manières» ou, plus simplement, «des manières» tout court. Cela signifie surtout, négativement, ne pas faire certaines choses, c'est-à-dire ne pas les faire comme les gens des classes inférieures. Le docteur Dubois de Poussière sur la ville sait que les notables de Macklin, même ceux qui sont sans le sou, « ne dînent pas au restaurant, surtout pas en compagnie de leurs épouses» (31).

Ces distances et ces distinctions de classe sont fréquemment brouillées, confuses. Elles varient selon les milieux et les régions. L'industriel québécois Victor Tremblay (Les vivants, les morts et les autres) est dérouté par la société de Montréal: elle est, 
constate-t-il, «encore mouvante dans ses structures au point où, si l'on excepte une minorité stable, elle donne l'impression de se renouveler presque chaque année... Les couches n'y sont pas étanches, même si l'on y distingue comme partout ailleurs des étages assez bien définis; on passe de l'un à l'autre sous cent prétextes» (76).

C'est pourquoi, plus encore que ces couches sociales aux paliers mobiles, ce qu'il importe d'identifier ce sont les postes dans la société qui sont les lieux de prestige réel, les lieux qui confèrent l'autorité et le pouvoir. L'autorité, le pouvoir, le prestige qui en dérive, varient eux-mêmes selon les milieux. Quel que soit le rayon de leur ampleur, ils sont associés à trois types principaux de structures sociales qui souvent s'entremêlent: les structures religieuses, politiques, économiques. Dans les gros villages et les petites villes, le maire et les marguilliers sont encore, avec le curé, les personnages-clefs (Fontile, Les Plouffe, Les vendeurs du temple). Le Bernard de La fin des songes de Robert Élie sait qu'un candidat peut difficilement être élu député sans l'appui de quatre «notables» de Rivière-Rouge: le maire, le notaire, le président de la Commission scolaire, le représentant des cultivateurs à la Coopérative (86). Le curé Loupret (Pierre le magnifique) se souvient que, du temps qu'il était jeune prêtre, il y a quarante ans, «les postes des villes étaient réservés aux prêtres ayant des relations influentes et leur rôle consistait surtout à transiger avec le Gouvernement» (41). Pierre Boisjoly lui-même sait fort bien ce qu'il fait lorsque, pour échapper à des ennemis qui le traquent, il revêt la soutane de son ami l'abbé Lippé. Avant l'arrivée du journaliste Bonneville dans Fontile, c'est le député et le maire qui contrôlaient le journal (98). On sait enfin le rôle tout-puissant que joue le procureur général dans l'odyssée rocambolesque de Pierre Boisjoly (Pierre le magnifique).

De plus en plus, cependant, ce sont les détenteurs du pouvoir économique qui sont mis en vedette. L'homme d'affaires-prêteur Arthur Prévost, de Poussière sur la ville, confie modestement au docteur Dubois qu'il «contrôle pas mal de choses à Macklin» et qu'il est dans les confidences de la banque (51). Le propriétaire d'une usine n'est pas important en tant que possesseur d'une entreprise qu'il dirige : ceci était la conception des générations passées, de la génération d'un Marius Tremblay (Les vivants, les morts et les autres). Il est important en tant que mandataire d'une puissance impersonnelle, sur-humaine, omniprésente: l'argent. Victor Tremblay, le fils et successeur de Marius, a fait sienne la maxime prononcée devant lui par un courtier de Montréal: "You must understand, money wants securities». L'argent, il en est convaincu, possède une existence morale, «comme la Royauté, le Gouvernement, en quelque manière indépendante de celui qui le détient et auquel elle survit» (233). Est-ce à cette forme de puissance que songeait Pierre Boisjoly (Pierre le magnifique) lorsqu'il rêvait de se lancer «d'un seul bond dans une lutte décisive avec les grandes forces qui dirigent la Province» (170) ? Pierre finit par se diriger vers le grand séminaire, mais d'autres héros de romans expriment une ambition analogue à celle qui l'a d'abord sollicité.

À ce point, il faut prêter attention à ce que disent nos romans des types d'ambition sociale chez les jeunes et des mécanismes de leur mobilité sociale. Par type d'ambition, j'entends une attitude vis-à-vis l'ensemble de la société. Ces attitudes 
peuvent se répartir selon quatre types principaux. Il y a les jeunes héros de romans qui acceptent la société telle qu'elle est et qui ambitionnent d'améliorer leur statut à l'intérieur des cadres existants. Il y a ceux qui ambitionnent de sortir de leur société ou, tout au moins, d'être d'abord appréciés et reconnus à l'étranger. Il y a les révoltés qui veulent se venger. Il y a enfin ceux qui ont décidé de transformer leur société. Au premier type d'ambition appartient le Jean Lévesque de Bonheur d'occasion, dont toute l'audace a comme objet de mettre «le pied sur le premier barreau de l'échelle» — et ensuite, "good-bye à Saint-Henri» (111). Un Denis Boucher et un Pierre Boisjoly alternent entre le second et le troisième types (Pierre le magnifique, 23, 68; Les Plouffe, 251, 253). À la quatrième catégorie appartient Maurice Tremblay, le licencié en sciences sociales de Les vivants, les morts et les autres qui, de militant et organisateur syndicaliste, passe au parti communiste.

En définitive, on peut ramener ces quatre types d'attitudes à deux principaux: l'attitude d'acceptation; l'attitude de contestation. Les mécanismes d'ascension sociale mis en œuvre par la première de ces deux attitudes sont familiers. C'est, comme dans le cas de Jean Lévesque, d'améliorer sa compétence technique; ou encore de nouer des relations utiles; ou encore de se tenir en état de disponibilité politique. L'occupation de journaliste, dans nos romans, constitue l'un des tremplins magiques facilitant ce type d'ascension. Quant à la seconde attitude, elle est conditionnée ou exprimée par l'une ou l'autre de deux activités privilégiées: celle d'écrivain et celle d'étudiant en sciences sociales. L'acte d'écrire, d'après nos romans, est le moyen par lequel l'individu non seulement prend ses distances avec la société mais la met en cause et, du fait même qu'il va la déconstruire et la reconstruire fictivement, s'installe dans une position de contestant permanent. L'autre activité, ai-je dit, est celle de l'étudiant en sciences sociales dont Maurice Tremblay, dans Les vivants, les morts et les autres, incarne un exemple dynamique. Il est fort probable que d'autres personnages de ce type apparaîtront avec plus de fréquence dans les romans à venir. Ils y sont d'ailleurs déjà, par l'intuition et l'exaltation, sous les traits de réformateurs ou de chefs syndicalistes. Ce Maurice Tremblay lui-même ne prétend-il pas qu'être «ouvrier» est "moins une condition sociale qu'un état d'esprit» (63)?

Soumettrons-nous, pour terminer, qu'il existe dans notre littérature une autre forme déguisée, symbolique, de contestation globale de la société que nous trouvons, par exemple, dans le Ashini d'Yves Thériault? Ashini dont le nom signifie « roc» et qui ambitionne d'être «l'ordonnateur d'une destinée nouvelle pour les (siens)» (51); Ashini le Montagnais qui demandait «que l'on rendît à ceux à qui on l'avait volée... la forêt sienne...» (57); Ashini qui, dans un rêve, est porté «vers des terres antiques où les Montagnais occupaient les faîtes et les hauts promontoires» (159-160). Le héros Ashini ne serait-il pas le symbole de la suprême contestation, de l'ambition de récupérer en une pleine possession et une parfaite autonomie une totalité sociale dont on a été dépossédé?... 


\section{CONCLUSION}

La société et les personnages que nous venons d'évoquer sont "les fantômes des vœux» de nos romanciers. Que pouvons-nous retenir de ces «rêveries»?

D'abord ceci, que dans nos romans, on se voit à n'en pas douter dans une société nettement définie et dont il est encore possible de faire le tour. Cette société est de plus d'une façon insulaire. Elle connaît ses frontières. Elle se tient repliée dans ces frontières. J'en prends comme preuve le fait que notre littérature ne fait mention d'aucun des univers sociaux d'une autre langue et d'une autre culture qui non seulement nous entourent mais sont mêlés à nous. Il n'y a d'exceptions à ce phénomène que Les sentiers de la nuit de Jean Simard et quelques romans d'Yves Thériault comme Aaron et Amour au goût de mer.

Il existe, par ailleurs, à l'intérieur de cette société, des distances et des décalages indéniables: entre les classes qui s'identifient avec de plus en plus de franchise; entre les générations qui se comprennent de moins en moins. Ce conflit entre générations, cette incompréhension entre pères et fils, reflètent-ils, comme l'a proposé Jeanne Lapointe, les drames profonds d'une classe moyenne qui a grandi trop vite et qui n'a pas eu le temps ou n'a pas su trouver les moyens de se mettre en situation ${ }^{3}$ Ou ne révèlent-ils pas aussi des cassures définitives dans notre continuité historique et des tentatives existentielles d'une nouvelle prise de possession d'un univers depuis longtemps désenchanté? Si le roman contemporain, au dire de Malraux, est une «signature», le roman canadien-français, pour sa part, atteste de bonnes volontés qui, au-delà de l'abdication mais encore en deçà de la tonifiante affirmation, sont engagées dans la difficile recherche de nouvelles raisons de vivre.

\section{BIBLIOGRAPHIE}

Bessette, Gérard (1960), Le libraire, Paris, René Julliard. Charbonneau, Robert (1945), Fontile, Montréal, Éditions de l'Arbre. Charbonneau, Robert (1941), Ils posséderont la terre, Montréal, Éditions de l'Arbre. ÉLIE, Robert (1950), La fin des songes, Montréal, Éditions Beauchemin. Gélinas, Pierre (1959), Les vivants, les morts et les autres, Montréal, Le Cercle du livre de France. Giroux, André (1948), Au delà des visages, Montréal, Les Éditions Variétés.

Giroux, André (1953), Le gouffre a toujours soif, Québec, Institut littéraire du Québec. Hamel, Émile-Charles (1962), Prix David, Montréal, Les Éditions de l'Homme. Langevin, André (1951), Évadé de la nuit, Montréal, Le Cercle du livre de France. Langevin, André (1953), Poussière sur la ville, Montréal, Le Cercle du livre de France. Langevin, André (1956), Le temps des hommes, Montréal, Le Cercle du livre de France. Lemelin, Roger (1944), Au pied de la Pente Douce, Montréal, Éditions de l'Arbre. Lemelin, Roger (1952), Pierre le magnifique, Québec, Institut littéraire du Québec. Lemelin, Roger (1948), Les Plouffe, Québec, Bélisle éditeur.

Lockquell, Clément (1949), Les élus que vous êtes, Montréal, Les Éditions Variétés. Marcotte, Gilles (1962), Le poids de Dieu, Paris, Flammarion.

3. Jeanne LAPOINTE (1961), A Few General Themes and Tendencies in French-Canadian Literature, The Duncan and John Gray Memorial Lecture, Toronto, 1er mars, texte inédit. 
Richard, Jean-Jules (1949), Neuf jours de haine, Montréal, Éditions de l'Arbre. Ringuet (Dr Philippe Panneton) (1938), Trente arpents, Paris, Flammarion.

Roy, Gabrielle (1954), Alexandre Chenevert, Montréal, Beauchemin, Le Cercle du livre de France. Roy, Gabrielle (1945), Bonheur d'occasion, Montréal, Société des Éditions Pascal, 2 vol. Simard, Jean (1956), Mon fils pourtant heureux, Montréal, Le Cercle du livre de France. Simard, Jean (1959), Les sentiers de la nuit, Montréal, Le Cercle du livre de France.

Thériault, Yves (1960), Aaron, Québec, Institut littéraire du Québec.

Thériault, Yves (1961), Amour au goût de mer, Montréal, Éditions Beauchemin.

Thériault, Yves (1960), Ashini, Montréal et Paris, Fides.

Thériault, Yves (1961), Cul-de-sac, Québec, Institut littéraire du Québec.

ThéRIAult, Yves (1951), Les vendeurs du temple, Québec, Institut littéraire du Québec.

Vaillancourt, Jean (1954), Les Canadiens errants, Montréal, Le Cercle du livre de France. 